\title{
Promoting Healthy Vision in Children Through the Use of Social Marketing
}

\author{
Danna Ethan ${ }^{*}$, Corey H Basch ${ }^{2}$ and Sonali Rajan ${ }^{3}$
}

${ }^{1}$ Department of Health Sciences, Lehman College, The City University of New York, New York, New York, USA ${ }^{2}$ Department of Public Health, William Paterson University, Wayne, New Jersey, USA

${ }^{3}$ Department of Health and Behavior Studies, Teachers College, Columbia University, New York, New York, USA

Over twenty percent of American school children have a vision problem, and this rate is markedly higher for children from lowerincome backgrounds [1]. The most common problem affecting vision is refractive error. Left untreated, refractive errors can worsen over time and may lead to more severe vision problems including amblyopia ("lazy eye"). Amblyopia is the leading cause of vision loss in children, however, this condition is correctable with early detection and prompt treatment $[2,3]$. In addition to possible vision loss, untreated vision problems can have a far-reaching effect on children, including emotional and developmental problems and even compromised professional productivity later in life [4]. In addition, researchers suggest that unaddressed vision problems may impact children's ability to learn effectively, which may prevent them from reaching their maximum academic potential [5]. Early detection, timely follow-up, and treatment are therefore essential in promoting healthy vision in children. Raising parents' awareness of this critically important child health issue should be addressed through a social marketing approach in concert with more established forms of health communication that are currently taking place.

Pediatric vision screenings are a critical springboard for identifying and treating children's vision problems. Identifying vision problems in children through screenings can be carried out by school nurses, pediatricians, trained laypeople, and vision care professionals. State and local policies vary widely with regard to vision screening and examinations for children. A small number of states have mandated eye exams for preschool children, while most states require that public schools provide basic vision screenings [4]. Follow-up when a problem is identified rests largely with the children's parents or guardians. For children who fail a vision screening and do seek followup vision care services outside of school, the most common form of treatment is prescription eyeglasses. Unfortunately, many children who need corrective lenses do not obtain them in a timely manner for various documented reasons including 1) associated financial costs, 2) transportation and scheduling conflicts related to vision care appointments, and 3) parents' perception that their child's potential vision problem is not a cause of great concern and thereby may lead to delayed or no action [6].

Reducing the prevalence of children's vision problems requires a comprehensive approach that should include federal, state, and locallevel coordinated efforts in the areas of legislation, policy, health care coverage, research, and education [4]. Communicating to parents about the critical importance of early detection and treatment of a possible vision problem has largely been the responsibility of schools, pediatricians, and vision care professionals. This message must reach more parents more often and in more ways. Extending health communication efforts beyond these realms to include a social marketing approach may increase parents' exposure to and potential action around this important child health issue.

As a primary recommendation in a report by the National Commission on Vision and Health, social marketing campaigns (via federally-funded grants to states) would deliver public health messages to parents (and relevant child health providers) geared towards early detection of children's vision problems [4]. A useful public health strategy, social marketing campaigns are used to disseminate health information in ways that appeal to a target audience with the aim of influencing health behaviors [7]. Similar to for-profit marketing techniques, effective social marketing involves assessing target populations' motivations and needs as well as the barriers that prevent positive behavior change [7].

An example of state-level social marketing efforts aimed towards parents includes Iowa's Department of Public Health's milk campaign, "Their Bodies Change. So Should their Milk," which encourages mothers of children age 2 and older to purchase $1 \%$ or fat-free milk [8]. Posters, television, and radio ads were developed after a needs assessment indicated the target population's desire for factual information with an emotional appeal [8]. To better identify autism spectrum disorders (ASDs) and other developmental disabilities in children, the Centers for Disease Control and Prevention's national "Learn the Signs. Act Early" campaign encourages parents to proactively recognize their children's developmental milestones and speak to a doctor if concerned [9]. Geared towards health care providers and educators as well as parents, the campaign utilizes an interactive website that houses an online informational video, a book parents can read to young children, milestone checklists, resource kit, and links to related websites.

Federal funding for social marketing efforts can bolster local needs assessments to determine parents' feelings, motivations and barriers that may prevent their follow-through with critical vision screening, prompt follow-up, and treatment for their children. Sufficient funding can also ensure appropriate evaluation that can be used to assess the effectiveness of planning and implementation measures. Utilization of social marketing by local and state health agencies as a means to educate and raise awareness about the key public health issue of children's vision can add meaningfully to a comprehensive, preventive approach. With the alarmingly high prevalence of vision problems in American children and the emphasis placed on narrowing the health and academic equity gap for high-risk youth, it is critical to plan, implement, and evaluate

*Corresponding author: Danna Ethan, Department of Health Sciences, Lehman College, The City University of New York 250 Bedford Park Boulevard West, Gillet Hall, Room 334, Bronx, New York 10468, P: 347-577-4034, USA, E-mail: danna.ethan@lehman.cuny.edu

Received September 04, 2013; Accepted September 05, 2013; Published September 06, 2013

Citation: Ethan D, Basch CH, Rajan S (2013) Promoting Healthy Vision in Children Through the Use of Social Marketing. J Mass Communicat Journalism 3: e140. doi:10.4172/2165-7912.1000e140

Copyright: ( 2013 Ethan D, et al. This is an open-access article distributed under the terms of the Creative Commons Attribution License, which permits unrestricted use, distribution, and reproduction in any medium, provided the original author and source are credited. 
Citation: Ethan D, Basch CH, Rajan S (2013) Promoting Healthy Vision in Children Through the Use of Social Marketing. J Mass Communicat Journalism 3: e140. doi:10.4172/2165-7912.1000e140

Page 2 of 2

innovative health communication strategies that inform and educate parents on the promotion of children's vision health.

\section{References}

1. Basch CE (2011) Vision and the achievement gap among urban minority youth Journal of School Health 81: 599-605.

2. http://www.aapos.org/terms/conditions/21.

3. http://www.pennmedicine.org/ophth/conditions/amblyopia.html.

4. Building a Comprehensive Child Vision Care System
5. Vaughn W, Maples WC, Hoenes R (2006) The association between vision quality of life and academics as measured by the College of Optometrists in Vision Development Quality of Life questionnaire. Optometry 77: 116-123.

6. Kimel L (2006) Lack of follow-up exams after failed school vision screenings: An investigation of contributing factors. The Journal of School Nursing 22: 156 162

7. http://publichealth.nc.gov/employees/socialmarketing.htm

8. http://www.idph.state.ia.us/INN/LowFatMilk.aspx

9. http://www.cdc.gov/ncbddd/actearly/index.html 